\title{
Abbreviations
}

HS The document or strand of the Pentateuch written by the Holiness School (also called the "H" source)

KTU Die keilalphabetischen Texte aus Ugarit. Edited by Manfried Dietrich, Oswald Loretz, and Joaquín Sanmartín. Münster: Ugarit-Verlag, 2013. 3rd enl. ed. of KTU: The Cuneiform Alphabetic Texts from Ugarit, Ras Ibn Hani, and Other Places. Edited by Manfried Dietrich, Oswald Loretz, and Joaquín Sanmartín. Münster: Ugarit-Verlag, 1995 (= CTU)

$\mathrm{P}$ Priestly strand of the Pentateuch

PT Priestly Torah strand of the Pentateuch

\section{Secondary Sources}

ANEP The Ancient Near East in Pictures Relating to the Old Testament. Edited by James B. Pritchard. 2nd ed. Princeton, NJ: Princeton University Press, 1994

ANET Ancient Near Eastern Texts Relating to the Old Testament. Edited by James B. Pritchard. 3rd ed. Princeton, NJ: Princeton University Press, 1969 Aramaic Papyri of the Fifth Century B.C. Edited by Arthur E. Cowley. Oxford: Clarendon, 1923

$A R A B \quad$ Ancient Records of Assyria and Babylonia. Daniel David Luckenbill. 2 vols. Chicago: University of Chicago Press, 1926-1927. Repr., New York: Greenwood, 1968 


$\begin{array}{ll}\text { ASOR } & \text { American Schools of Oriental Research } \\ \text { ATD } & \text { Das Alte Testament Deutsch } \\ \text { AYB } & \text { Anchor Yale Bible } \\ \text { BAR } & \text { Biblical Archaeology Review } \\ \text { BBB } & \text { Bonner biblische Beiträge } \\ \text { BDB } & \text { Brown, Francis, S. R. Driver, and Charles A. Briggs. } \\ & \text { A Hebrew and English Lexicon of the Old Testament }\end{array}$

BETL Bibliotheca Ephemeridum Theologicarum Lovaniensium BHS Biblia Hebraica Stuttgartensia. Edited by Karl Elliger and Wilhelm Rudolph. Stuttgart: Deutsche Bibelgesellschaft, 1983

BZAW Beihefte zur Zeitschrift für die alttestamentliche Wissenschaft

CBQ Catholic Biblical Quarterly

$C D$

Church Dogmatics by Karl Barth. Translated and edited by G. W. Bromiley, T. F. Torrance, and G. T. Thompson. 14 vols. Edinburgh: T\&T Clark, 1956-1975. Standard citation format references volume number, number of part of the volume, and section and page number.

COS The Context of Scripture. Edited by William W. Hallo. 3 vols. Leiden: Brill, 1997-2002

CW $\quad$ Collected Works of C. G. (Carl Gustav) Jung. Edited by Herbert Read, Michael Fordham, and Gerhard Adler. Bollingen series 20. 20 vols. New York: Pantheon, 1953

FAT Forschungen zum Alten Testament

FOTL Forms of the Old Testament Literature

GKC Gesenius' Hebrew Grammar. Edited by Emil Kautzsch. Translated by A. E. Cowley. 2nd ed. Oxford: Clarendon, 1910

HALOT The Hebrew and Aramaic Lexicon of the Old Testament. Ludwig Koehler, Walter Baumgartner, and Johann J. Stamm. Translated and edited under the supervision of Mervyn E. J. Richardson. 4 vols. Leiden: Brill, 1994-1999

HAT Handbuch zum Alten Testament

HSM Harvard Semitic Monographs $I B$

IBHS An Introduction to Biblical Hebrew Syntax. Bruce K. Waltke and Michael O'Connor. Winona Lake, IN: Eisenbrauns, 1990 


\begin{tabular}{|c|c|}
\hline JAOS & Journal of the American Oriental Society \\
\hline$J B L$ & Journal of Biblical Literature \\
\hline Joüon & $\begin{array}{l}\text { Joüon, Paul. A Grammar of Biblical Hebrew. Translated and } \\
\text { revised by T. Muraoka. } 2 \text { vols. Rome: Pontifical Biblical } \\
\text { Institute, } 1991\end{array}$ \\
\hline$S O T$ & Journal for the Study of the Old Testament \\
\hline OTSup & $\begin{array}{l}\text { Journal for the Study of the Old Testament Supplement } \\
\text { Series }\end{array}$ \\
\hline JSPSup & $\begin{array}{l}\text { Journal for the Study of the Pseudepigrapha Supplement } \\
\text { Series }\end{array}$ \\
\hline KBL & $\begin{array}{l}\text { Koehler, Ludwig, and Walter Baumgartner. Lexicon in Veteris } \\
\text { Testamenti libros. 2nd ed. Leiden: Brill, } 1958\end{array}$ \\
\hline LHBOTS & The Library of Hebrew Bible/Old Testament Studies \\
\hline NDERF & $\begin{array}{l}\text { Stories collected at the Near Death Experience Research } \\
\text { Foundation, http://www.nderf.org/index.htm }\end{array}$ \\
\hline$N I B$ & $\begin{array}{l}\text { The New Interpreter's Bible. Edited by Leander E. Keck. } \\
12 \text { vols. Nashville: Abingdon, 1994-2004 }\end{array}$ \\
\hline NIBCOT & $\begin{array}{l}\text { New International Biblical Commentary on the Old } \\
\text { Testament }\end{array}$ \\
\hline NICOT & New International Commentary on the Old Testament \\
\hline OTL & Old Testament Library \\
\hline OtSt & Oudtestamentische Studiën \\
\hline PTMS & Princeton Theological Monograph Series \\
\hline$R B$ & Revue biblique \\
\hline SBLStBL & Society of Biblical Literature Studies in Biblical Literature \\
\hline SBLSymS & Society of Biblical Literature Symposium Series \\
\hline SCS & $\begin{array}{l}\text { Society of Biblical Literature Septuagint and Cognate } \\
\text { Studies }\end{array}$ \\
\hline StBibLit & Studies in Biblical Literature (Lang) \\
\hline TOTC & Tyndale Old Testament Commentaries \\
\hline UCOP & University of Cambridge Oriental Publications \\
\hline$U T$ & $\begin{array}{l}\text { Ugaritic Textbook. Cyrus H. Gordon. Analecta Orientalia } 38 . \\
\text { Rome: Pontifical Biblical Institute, } 1965\end{array}$ \\
\hline$V T$ & Vetus Testamentum \\
\hline VTSup & Supplements to Vetus Testamentum \\
\hline WBC & Word Biblical Commentary \\
\hline WMANT & $\begin{array}{l}\text { Wissenschaftliche Monographien zum Alten und Neuen } \\
\text { Testament }\end{array}$ \\
\hline
\end{tabular}




\section{Textual Witnesses}

LXX Septuagint

MT Masoretic Text

\section{Greek and Latin Authors}

Ambrose

$$
\text { Fid. De fide }
$$

Augustine

$$
\begin{array}{ll}
\text { Civ. } & \text { The City of God (De civitate Dei) } \\
\text { Conf. } & \text { Confessions }
\end{array}
$$

Herodotus

$$
\text { Hist. Histories (Historiae) }
$$

Josephus

$$
\text { Ant. Jewish Antiquities }
$$

Plato

$$
\text { Phaed. Phaedo }
$$

Theodoret of Cyrus

Ezech. Commentary on the Prophet Ezekiel

\section{Traditional Jewish Texts and Scholars}

Abarbanel Don Isaac Abarbanel (Abravanel). Iberian Peninsula-Italy, 1437-1508

b. 'Arak. Babylonian Talmud: Tractate 'Arakin

b. Ber. Babylonian Talmud: Tractate Berakot

b. Sanh. Babylonian Talmud: Tractate Sanhedrin

Eliezer of Jewish French exegete, Northern France, twelfth

Beaugency century CE

Ibn Ezra Rabbi Abraham Ibn Ezra, 1089-1167, Spanish-born biblical scholar and philosopher

Malbim Acronym of Rabbi Meïr Loeb ben Jehiel Michel Wisser, Eastern Central Europe, 1809-1879

Meșudat David One part of a two-tiered Bible commentary by David and Jehiel (son of David) Altschuler, Galicia, Eastern Europe, eighteenth century CE 


$\begin{array}{ll}\text { m. Mid. } & \text { Mishnah: Tractate Middot } \\ \text { m. Yoma } & \text { Mishnah: Tractate Yoma } \\ \text { m. Zebah } & \text { Mishnah: Tractate Zebahim } \\ \text { Radak } & \begin{array}{l}\text { Acronym of Rabbi David Kimhi (Kimhi), Provence, } \\ \text { southern France, ca. 1160-1235 }\end{array} \\ \text { Ramban } & \begin{array}{l}\text { Acronym of Nahmanides, Catalonia, Spain, 1194-1270 } \\ \text { Rashbam }\end{array} \\ & \begin{array}{l}\text { Acronym of Rabbi Samuel ben Meir, Troyes, northern } \\ \text { France, ca. 1085-1158, grandson of Rashi }\end{array} \\ \text { Rashbi } & \begin{array}{l}\text { Acronym of Rabbi Simeon bar Yochai, second century CE, } \\ \text { Tannaitic sage, Israel, disciple of Rabbi Akiva }\end{array} \\ \text { Rashi } & \text { Acronym of Rabbi Solomon ben Isaac, Troyes, northern } \\ & \text { France, 1040-1105 }\end{array}$

\section{Modern English Bible Translations}

$\begin{array}{ll}\text { ASV } & \text { American Standard Version (Thomas Nelson) } \\ \text { BBE } & \text { Bible in Basic English (S. H. Hooke, 1949) } \\ \text { CEB } & \text { Common English Bible (Abingdon) } \\ \text { CEV } & \text { Contemporary English Version (American Bible Society) } \\ \text { CJB } & \text { Complete Jewish Bible (Lederer Foundation) } \\ \text { ESV } & \text { English Standard Version (Crossway) } \\ \text { GNT } & \text { Good News Translation (American Bible Society) } \\ \text { KJV } & \text { King James Version (The Queen's Printer, Cambridge } \\ & \text { University Press) } \\ \text { LITV } & \text { Green's Literal Translation of the Bible (Sovereign Grace } \\ & \text { Publishers) } \\ \text { MESSAGE } & \text { The Message (Eugene Peterson) } \\ \text { NABR } & \text { New American Bible, Revised (Confraternity of Christian } \\ & \text { Doctrine) } \\ \text { NASB } & \text { New American Standard Bible (Lockman) } \\ \text { NEB } & \text { New English Bible (Oxford University Press) } \\ \text { NET } & \text { New English Translation (Bible.org) } \\ \text { NETS } & \text { A New English Translation of the Septuagint (Oxford Univer- } \\ & \text { sity Press) } \\ \text { NIV } & \text { New International Version (Zondervan) } \\ \text { NJB } & \text { New Jerusalem Bible (Darton, Longman, \& Todd, and } \\ & \text { Doubleday) } \\ \text { NJPS } & \text { Tanakh (Jewish Publication Society) }\end{array}$




$\begin{array}{ll}\text { NKJV } & \text { New Kings James Version (Thomas Nelson) } \\ \text { NLT } & \text { New Living Translation (Tyndale) } \\ \text { NRSV } & \text { New Revised Standard Version (National Council of the } \\ & \text { Churches of Christ) } \\ \text { REB } & \text { Revised English Bible (Oxford University Press) } \\ \text { RSV } & \text { Revised Standard Version (National Council of the } \\ & \text { Churches of Christ) } \\ \text { VOICE } & \text { The Voice Bible (Thomas Nelson) } \\ \text { YLT } & \text { Young's Literal Translation (1898; Public Domain) }\end{array}$

